SSM-D-08-01352-R1

This is the version of the manuscript as accepted after peer-review but prior to any changes made during the proofing and publication process. It is made available under a Creative Commons Attribution Non-Commercial No Derivatives Licence. The full published version is available from Elsevier at: http://dx.doi.org/10.1016/j.socscimed.2009.06.012

\title{
Demographic data in asthma clinical trials: A systematic review with implications for generalizing trial findings and tackling health disparities
}

\section{Geoff K. Frampton}

Southampton Health Technology Assessments Centre (SHTAC), School of Medicine, University of Southampton, Epsilon House, Southampton Science Park, Chilworth, Southampton, Hampshire SO16 7NS, UK

\section{Jonathan Shepherd}

Southampton Health Technology Assessments Centre (SHTAC), School of Medicine, University of Southampton, Epsilon House, Southampton Science Park, Chilworth, Southampton, Hampshire SO16 $7 \mathrm{NS}, \mathrm{UK}$

\section{Jean-Lou C.M. Dorne}

Institute of Human Nutrition, School of Medicine, University of Southampton, UK

Present address: European Food Safety Authority, Largo N Palli 5/A, 43100

Parma, Italy.

\begin{abstract}
The prevalence of asthma, and the morbidity, adverse events, mortality and healthcare utilisation of asthmatic patients vary widely among racial/ethnic and other socio-demographic groups. Debates over the meanings of race and ethnicity and the strategic need to resolve health inequalities have prompted extensive recommendations for reporting and analyzing racial/ethnic and demographic information in clinical trials. We conducted a systematic review to determine the extent to which race/ethnicity, socioeconomic status and other demographic variables are analyzed and reported in publications from randomized controlled trials of asthma interventions. Randomized controlled trials of inhaled corticosteroids and long-acting b-agonists in asthmatic patients were identified by systematically searching 12 electronic bibliographic databases. We identified peer-reviewed papers reporting 87 relevant trials published during 1985-2006, from which we extracted data on patients' race/ethnicity, ancestry, gender, socio-economic variables and geographical attributes. The proportion of the papers reporting the race/ ethnicity of their participants was lower than would be expected by chance and has
\end{abstract}


recently declined. None of the papers included race/ethnicity or gender in statistical analyses or reported socio-economic variables, ancestry, or genetic data for their participants, and few discussed the generalizability of their findings. The frequency of reporting race/ethnicity was statistically significantly lower in trials conducted in the UK than in the US, but 23 of the 87 papers did not identify countries. Despite extensive recommendations in the literature, guidance from health agencies on analyzing and reporting demographic data in clinical trials still appears inconsistent and vague. There remains a need to improve guidance on the representation and analysis of minority populations in asthma clinical trials, in order to encourage transparent reporting of population selection, analysis approaches, and trial generalizability. To assist this process, asthma clinical trials should be based on clear hypotheses that link both to existing demographic evidence and to demographic healthcare goals.

Keywords: Ethnic groups; Health status disparities; Minority health; Pharmacogenetics; Socioeconomic factors; Information dissemination; Systematic review

\section{Introduction}

The prevalence of asthma, and asthma-related morbidity, mortality and hospitalization rates are wellknown to differ between population groups that have different ancestral, geographical, and/or cultural backgrounds, often referred to as 'racial', 'ethnic' or 'racial/ ethnic' groups (130 examples from 86 publications are given in Supplementary Table S1).There is extensive evidence that such racial/ ethnic differences in asthma may be confounded with, interact with, or serve as a proxy for effects of physiological variables such as lung function, and socio-economic variables such as income, education, exposure to pollutants, exposure and sensitization to allergens, and access to healthcare (72 examples from 42 publications are given in Supplementary Table S2). Asthma severity has been linked, for example, with social factors that differ among racial/ethnic groups, including neighbourhood violence (Tonorezos et al., 2008) and patterns of communication between doctors and patients (Diette \& Rand, 2007). Interaction ofmany biological, social and environmental factors can influence the treatment received by asthma patients and their adherence to it (Shanawani, 2006). The prevalence of asthma and the timing of asthma onset are influenced by patterns of migration which in turn differ among racial/ethnic groups. For example, immigrants often have lower prevalence of asthma than indigenous populations (Kuehni, Strippoli, Low, \& Silverman, 2007; Migliore et al., 2007; Netuveli et al., 2005), and asthma prevalence varies with age at migration (Kuehni et al., 2007), place of origin (Holguin et al., 2005; Tedeschi, Barcella, Bo, \& Miadonna, 2003), and duration of residence at the destination (Leung, Carlin, Burdon, \& Czarny,1994;Migliore et al., 2007). Asthma incidence, prevalence and risk of exacerbations also vary with age and gender, being higher in boys than girls before puberty and then, in adolescents and adults, higher in women than men (Johnston \& Sears, 2006). Gender differences in the incidence and prevalence of asthma have been documented in publications, surveys and databases for several decades (Almqvist, Worm, \& Leynaert, 
2008), although there appears to have been limited public awareness of this (Jensen-Jarolim \& Untersmayr, 2008).Recent studies have showngender differences in the efficacy of some asthma drugs (Johnston et al., 2007; Szefler et al., 2005) and in the risks of adverse events (Ishizuka et al., 2007), suggesting that effects of gender should be considered in asthma clinical studies (de Benedictis, Baraldi, \& Boner, 2008).

Studies of genetic polymorphisms have highlighted the potential value of reporting genetic information for populations in clinical trials (Choudhry et al., 2005; Salari et al., 2005). Several gene polymorphisms are relevant in asthma patients as they influence responses to b-agonists, glucocorticosteroids and leukotriene modifier drugs, which are the three main classes of asthma medication (Sayers \& Hall, 2007). Polymorphism in the b2-adrenergic receptor gene (ADRB2) differs between African American, Caucasian and Chinese populations (Xie et al., 1999) and other racial/ethnic groups (Maxwell et al., 2005; Munakata et al., 2006), and has been related to variation in patients' responsiveness to long-acting b-agonists (Israel et al., 2004; Wechsler et al., 2006), shortacting b-agonists (Hall \& Sayers, 2007; Israel et al., 2000), and the frequency of asthma exacerbations (Taylor et al., 2000). Racial/ethnic differences have also been observed in the elimination of the longacting b-agonist bambuterol (Koysooko et al., 1987) and would be expected in the clearance of inhaled corticosteroids such as budesonide, ciclesonide and fluticasone. These drugs are metabolised in the liver and gut by the cytochrome P450 enzyme CYP3A4 (Nave, Fisher, \& McCracken, in press; Pearce, Leeder, \& Kearns, 2006; Peet, Enos, Nave, Zech, \& Hall, 2005), whose activity varies threefold between some racial/ethnic groups (Dorne, 2007). Such genetic variation in pharmacokinetics raises the prospect of optimising asthma management for efficacy and safety using pharmacogenetic approaches, but there is a shortage of relevant studies (Hall \& Sayers, 2007).

The relative importance of and interactions among race/ethnicity, socio-economic status and genetics for asthma is the subject of considerable debate (e.g., Apter, 2006; Cooper, 2003; Cooper, Kaufman, $\&$ Ward, 2003). The latest guidelines from the Global Strategy for Asthma Management and Prevention (Bateman et al., 2008) acknowledge, somewhat simplistically, that the apparent 'racial' and 'ethnic' differences in the prevalence of asthma reflect 'underlying genetic variances with a significant overlay of socio-economic and environmental factors'. It is clear that research into asthma should take these population differences into consideration but there has been considerable confusion, inconsistency and disagreement over the meaning and appropriateness of terms such as 'race', 'ethnicity' and 'race/ethnicity' (Smart, Tutton, Martin, Ellison, \& Ashcroft, 2008). The concept of 'race' in humans has been discredited by the American Anthropological Association (AAA) and others as a social construct that has limited biological meaning (Agyemang, Bhopal, \& Bruijnzeels, 2005; American Anthropological Association, 1997, Anonymous, 2000). Although 'ethnicity' is inconsistently defined (Anonymous, 2000; Collins, 2004), it usually avoids the discredited notion of 
human sub-species and the potential for pejorative racist interpretation that has been associated with the term 'race' (Huth, 1995; Witzig, 1996). 'Ethnicity' usually implies shared origins, a shared social background, and/or shared social culture and traditions, including language and religion (Agyemang et al., 2005; Bhopal, 2004; Senior \& Bhopal, 1994; Witzig, 1996). The AAA proposed the term 'race/ethnicity' in 1997 as a means to facilitate gradual elimination of 'race' from the US population census (American Anthropological Association, 1997) and 'race/ethnicity' has since been widely adopted (Bhopal, 2004). Here, we use 'race/ethnicity' to refer broadly to any variables that might be classed as 'racial' or 'ethnic', as defined by Bhopal (2004).

There has been extensive criticism of the use in medical research of racial/ethnic descriptions such as Asian (Bhopal, Phillimore, \& Kohli, 1991), Black (Agyemang et al., 2005), Caucasian (Bhopal \& Donaldson, 1998), and Latino (Gonza' lez Buchard et al., 2005) because differences in ancestry and migration which might have clinical relevance cannot be resolved at these very broad, usually undefined, classes. Others, however, have argued that the use of broad racial/ethnic categories may have some merit as proxy variables that, in the absence of more refined technology, may encode important information as a result of their role in social stratification (Cohn, 2006; Kaufman \& Cooper, 2001). Further confusion about the value of racial/ethnic groups has been caused by a general failure to clearly define such groups, inconsistent interpretation of the same concept by different people, uncertainty about the comparability of self-assigned and independently assigned racial/ethnic status, and an increase in the number of people with multi-ethnic backgrounds for whom no clear classification system exists (Witzig, 1996). These difficulties with racial/ethnic classifications have led to numerous recommendations in the medical literature that broad racial/ethnic classes such as Asian, Black, Caucasian, Hispanic, Latino, or White should be abandoned in favour of, or at least supplemented by, more precise demographic information including ancestry, migration history, culture and socio-economic variables (e.g., inter alia, Agyemang et al., 2005; Anonymous, 1996; Bhopal, 2004; Bhopal \& Donaldson, 1998; Coons, 2006; Huth, 1995; International Committee of Medical Journal Editors, 1997; Kaplan \& Bennett, 2003; Winker, 2004). Despite these recommendations, broad racial/ethnic classifications continue in clinical research (Schwartz, 2001) and in epidemiology and public health (Agyemang, 2006).

Asthma research studies need to consider the importance of population differences in asthma morbidity, mortality, responses to management, adverse events, access to healthcare, and opportunities for pharmacogenetic therapy, whilst taking into account the difficulties of recruiting, including and describing relevant population groups in clinical trials. The representation of racial/ethnic minorities in clinical trials is an important component of strategies for reducing inequalities in healthcare (Committee on Understanding and Eliminating Racial and Ethnic Disparities in Health Care, 2003), and a recognised aim of health agencies including the US National Institutes of 
Health (National Institutes of Health, 2004) and the UK National Health Service (Hussain-Gambles, Atkin, \& Leese, 2004). Our objective was to clarify the extent to which randomized controlled clinical trials of inhaled asthma interventions consider the ancestry, genetic profiles, geography, and socioeconomic status of their populations for establishing the generalizability of the trials (i.e. to which populations their results are applicable) (Hussain-Gambles et al., 2004), for providing patient-centred clinical guidance, and for supporting pharmacogenetic developments in asthma management. As gender differences in asthma have recently attracted renewed interest (Almqvist et al., 2008; de Benedictis et al., 2008; Jensen-Jarolim \& Untersmayr, 2008), we also sought to clarify the extent to which gender was reported and analyzed in the asthma clinical trials.

\section{Methods}

Information was obtained from two peer-reviewed systematic reviews of randomized controlled trials which evaluated the clinical efficacy and safety of inhaled corticosteroids and long-acting betaagonists for asthma treatment in adults (Shepherd et al., 2008) and children (Main et al., 2008). The reviews were conducted to inform clinical guidance on asthma management that were being updated by the UK National Institute for Health and Clinical Excellence (UK National Institute for Health and Clinical Excellence, 2008a, 2008b). The systematic reviewwe report here is a secondary extraction of data on race/ethnicity and other demographic variables from the papers that were included in these primary reviews. Inclusion criteria for the primary systematic reviews were: populations: patients of any age, gender, or nationality with chronic asthma of any severity, but excluding those with chronic obstructive pulmonary disease and other respiratory co-morbidities. Interventions: five inhaled corticosteroids and two combination drugs (inhaled corticosteroid plus long-acting b-agonist) (Main et al., 2008; Shepherd et al., 2008), compared head-to-head in any combination or against a placebo, at any of their approved doses, with a minimum intervention duration of four weeks. Design: randomized controlled trials. Outcomes: trials were included if they reported quantitative data for at least one of the following outcomes: forced expiratory volume in one second; am peak expiratory flow; pm peak expiratory flow; nocturnal awakening; symptom-free days; symptom-free nights; symptom scores; health-related quality of life scores; rescue medication use; exacerbations; adverse events. A search strategy common to both reviews was used to identify relevant studies (Supplementary Table S3). Twelve electronic bibliographic databases were searched during January to March 2006, with searches updated (re-run) in September 2006 (Supplementary Table S3). Further details of the reviews are given in the published reports (Main et al., 2008; Shepherd et al., 2008), which tabulate the populations, patient numbers, baseline characteristics, interventions, outcomes, data extraction, and assessments of quality for each of the trials. In total, 87 randomized controlled trials were included (Fig. 1). 
We noticed that these 87 randomized controlled trials appeared to provide little if any information on race/ethnicity and socioeconomic status. To investigate the reporting of race/ethnicity and related variables in more detail, we extracted from the 87 trials all available data on race/ethnicity, any socioeconomic variables (e.g., income, place of residence, social class, religion), and demographic aspects of the trials (e.g., the number, location and identity of the countries involved) using a standard data template (Supplementary Table S4). The data were extracted by one reviewer and checked independently by another reviewer.

The 87 randomized controlled trials included in our systematic review (Main et al., 2008; Shepherd et al., 2008) comprised 70 on adults (patients aged 12 years or above), 16 on children (aged less than 12), and one on both adults and children (Fig. 1).

Of the 87 trials, 23 (26\%) stated the race/ethnicity of their participants (Supplementary Table S4). This is a significantly lower proportion than would be expected by chance $(\mathrm{p}<0.001$; binomial test), assuming that the authors had an equal likelihood of either reporting or not reporting this variable. Of these 23 trials, none explained how race/ethnicity was assigned (whether by self-report or investigator classification) and none provided quantitative analyses of race/ethnicity data or discussed the possible implications for clinical practice of the racial/ethnic composition of their patient populations. The number of randomized controlled trials published per year has increased but the proportion reporting race/ethnicity has declined; only 1/14 trials reported race/ethnicity in 2006 (Fig. 2). Overall, the proportions of trials that reported race/ethnicity (Supplementary Table S4) did not differ significantly between studies of adults (21/70) and children $(2 / 16)(\mathrm{p}=0.215$; Fisher's Exact test).

Trials that reported race/ethnicity all referred to broad racial/ethnic groups without providing more precise information on ancestry, demographics or migration (Supplementary Table S4). None of the 87 trials reported genetic information for the included populations. Of the 23 trials that reported race/ethnicity, six were population-specific, being conducted on patients who were all from one broad racial/ethnic group, described as Caucasian (two trials), Indian (two trials), Chinese (one trial), or Italian (one trial). The remaining 17 trials reported baseline information for multiple racial/ ethnic groups but did not subsequently refer to this information when analyzing and interpreting outcomes. Where participants were described as 'Caucasian' (10 trials) or 'Black' ( 6 trials), the Caucasian participants consistently occupied $80 \%$ of the study population, whereas the Black participants occupied $23 \%$. Socioeconomic variables such as household income and place of residence were not reported in any of the 87 trials. One trial (number 60 in Supplementary Table S4) identified the religion of its participants (Hindu, Muslim, Christian) but did not analyze this information. 
The trials were conducted in at least 50 countries, with the number of countries per trial ranging from 1 to at least 22 and the number of centres per trial from 1 to at least 246. 'Centres' comprised hospitals, departments, clinics, physicians, or were not described. Countries were named in 38 trials, could be inferred indirectly in a further 26 , but were not identified in the remaining 23 trials (Supplementary Table S4).

Forty-one (47\%) of the trials were multi-national. Of these, 31 stated that country was included as a factor or co-variable in statistical analyses of intervention effects, or that the mean data for certain clinical outcomes were adjusted for country. However, none of the trials explained these analyses or adjustments, and only four mentioned, without any empirical data, that there were no significant differences in clinical outcomes between countries, or no country-by-intervention interactions (Supplementary Table S4). The majority (38) of the 41 multi-national trials did not mention the generalizability of their findings. Authors of three trials (numbers 49, 64 and 81 in Supplementary Table S4) stated that their results would be valid in several countries but did not say which countries, or for which clinical outcomes.

Of 46 trials that were country-specific, 7 were conducted exclusively in the US (trials 26, 39, 40, 47, 53, 55, and 79 in Supplementary Table S4) and9 exclusively in the UK (trials1, 9,10,13,19, 21, 27, 30, 33 in Supplementary Table S4). No other countries had more than four country-specific trials. The proportion of trials that reported race/ethnicity differed significantly between those conducted in the US (6/7) and UK (1/9) ( $\mathrm{p}=0.009$; Fisher's Exact test).

Gender was reported at baseline for nearly all (85/87) of the randomized controlled trials. The percentage of males ranged from $18 \%$ to $93 \%$ (mean $50.4 \%$, median $44.5 \%$, mode $43 \%$ ). However, only three of the trials mentioned gender when discussing outcomes (studies 3, 14 and 30 in Supplementary Table S4). These referred to a single female withdrawal (study 3), an observation that patients with high morning serum cortisol concentrations (>7.0 $\mathrm{mmol} \mathrm{l}^{-1}$ ) were all women (study 14), and an observation that proportionally more women in one intervention completed the study (study $30)$.

\section{Discussion}

Our systematic review suggests that the proportion of asthma clinical trials reporting race/ethnicity (defined in its broadest sense to include any aspects of race and/or ethnicity) has recently declined. This is in contrast to the findings of a review by Brahan and Bauchner (2005) which found an increase in the proportion of papers that reported race/ethnicity for paediatric asthma clinical trials. Brahan and Bauchner (2005) examined five pre-selected general medical journals for original research articles on 
paediatric asthma published during 1991-1993 and 2000-2002, not limited to randomized controlled trials. However, they included only studies with US researchers and institutions, which are more likely than European studies to report race/ethnicity (Sheikh, Panesar, Lasserson, \& Netuveli, 2004). Our review represents the most comprehensive evaluation carried out so far on the reporting and analysis of demographic information in asthma clinical trials, and included studies published in 32 English language journals without a priori restrictions on the nationality of researchers or type of publication (Main et al., 2008; Shepherd et al., 2008). Other reviews have investigated the reporting of race/ethnicity for non-asthmatic populations, for example Corbie-Smith, St George, Moody-Ayers, and Ransohoff (2003) surveyed three high-impact medical journals and evaluated primary research articles published on diabetes, cardiovascular disease, cancer and HIV/AIDS during 1989-2000. Walsh and Ross (2003) surveyed three general medical journals and evaluated all original primary research articles published during 1999-2000, but limited their evaluation to studies with US researchers. Ma, Khan, Kang, Zalunardo, and Palepu (2007) surveyed four high-impact general medical journals and evaluated all primary research articles published during 1999-2003 that reported race/ethnicity. The two reviews that compared reporting and non-reporting of race/ethnicity indicated that $63.5 \%$ (Walsh \& Ross, 2003) or 59\% of publications (Corbie-Smith et al., 2003) had reported race/ethnicity during the most recent time periods they surveyed. These are higher frequencies of reporting race/ethnicity than we found for the asthma trials, but might not be directly comparable with our results due to differences in methodology.

Our systematic review differed from the others, for example, in that it was limited to randomized controlled trials but was not restricted to a small subset of pre-specified journals. Our finding that none of the asthma clinical trials explained how they assigned race/ethnicity classes to their patients (e.g., whether self-reported or researcher-assigned) is broadly consistent with results of a study by Shanawani, Dame, Schwartz, and Cook-Deegan (2006) which showed that the majority of medical research articles claiming associations among genotype, outcome and race/ethnicity did not explain their methods for assigning race or ethnicity.

With such a wealth of historical evidence that asthma diagnosis, morbidity, mortality, treatment, and healthcare resource utilisation differ with race/ethnicity and/or socio-economic status (Supplementary Tables S1 and S2), together with the numerous reporting recommendations for authors, it seems surprising that race/ethnicity and socio-economic variables are rarely reported and analyzed in randomized controlled trials. The higher frequency of reporting race/ethnicity in the US than in the UK is consistent with findings of a review by Sheikh et al. (2004) and may reflect the fact that government-funded research programmes are contractually required in the US, but not in Europe, to provide statistics on race/ethnicity. Sheikh et al. (2004) urged European governments and respiratory 
agencies to consider the US model, but noted that even in the US fewer than $65 \%$ of recent asthma clinical trials had reported race/ethnicity.

Gender differences in asthma incidence and prevalence have been observed for decades (Almqvist et al., 2008), but clinical trials are only now starting to demonstrate the potential importance of gender differences in asthma drug efficacy (Johnston et al., 2007; Szefler et al., 2005) and adverse events (Ishizuka et al., 2007). Compared to race/ethnicity and socio-economic status, there appears to have been less awareness of, or concern about gender differences in asthma (Jensen-Jarolim \& Untersmayr, 2008), which might explain why only three of the 87 randomized controlled trials that we evaluated mentioned gender when discussing their outcomes. The gender differences in post-treatment serum cortisol concentrations and in the number of patients who completed a study (mentioned in studies 14 and 30, respectively; Supplementary Table S4), together with the gender differences in asthma drug efficacy and adverse events observed in other studies, suggest that gender differences in asthma management and outcomes should be considered more critically in future asthma clinical trials.

\section{Limitations and strengths of the review}

Our review was conducted during March to September 2006, hence trials published since then are not included. However, it is unlikely that reporting of race/ethnicity would have improved since then, as we are unaware of any obvious new recommendations or policies to stimulate such a change. As our review focuses on asthma, our findings might not reflect the reporting of race/ethnicity for other conditions. Our findings are important, however, because of the high prevalence of asthma in developed countries and its extensive association with racial/ethnic and other demographic inequalities in health outcomes and treatment (Supplementary Tables S1 and S2).

Our review does not rule out the possibility that some authors might have recruited minority populations but failed to report the details. We assume that any authors who had gone to the trouble of recruiting minority populations would have indicated so. Our findings suggest that there is very limited recruitment of minority groups in asthma clinical trials, but it is also clear that trial reporting was inadequate, as in the majority (74\%) of the trials the racial/ethnic groups that were included were not identifiable.

Key strengths of our revieware that it was systematic, impartial, and used the same evidence base from the published literature as used by the UK National Institute for Health and Clinical Excellence (NICE) to underpin their clinical guidance on asthma management. Our findings provide an insight into the availability to NICE and other health agencies of information from the published literature on race/ethnicity and other demographic variables. 
Numerous difficulties in recruiting minority groups to clinical trials have been reported, for example, healthcare professionals appear to be unaware of the importance of representational sampling of populations (Hussain-Gambles et al., 2004). Researchers may also be discouraged from including minority populations by issues of ethics, language difficulties, unclear classification and definition of minority groups, health provider attitudes, religious standards and cultural modesty (Hussain-Gambles et al., 2004; Sheikh et al., 2004), as well as by institutional racism, other political sensitivities (Aldhous, 2002; Witzig, 1996), and arguments that race/ethnicity should not be included in studies unless there is a biological, scientific, or sociological reason for doing so (Rivara \& Finberg, 2001).

Even if minority populations are recruited to trials, word limits imposed by journals might discourage authors from reporting all the available race/ethnicity and socio-economic information, especially as authors are already under pressure from CONSORT guidance to report numerous other variables. CONSORT guidance does not explicitly require reporting or analysis of race/ethnicity (Moher, Schultz, \& Altman, 2001), although Sheikh et al. (2004) argued that it should. According to a recent survey, only $6 \%$ of articles sampled in three US paediatric journals reported race/ethnicity to comply with journals' peer-review requirements (Walsh \& Ross, 2003). Online data supplements and electronic journal articles are possible options for improving the publication of demographic information from clinical trials, provided that editorial and peer-review requirements for the provision of such data are enforced.

Generalizing the results - but to which populations and patients?

Under-representation of ethnic minority populations in clinical trials may seriously compromise the generalizability of the findings (Cohn, 2006; Hussain-Gambles et al., 2004). According to research governance guidance, the body of research evidence must reflect population diversity (Allmark, 2004), and this is particularly relevant for asthma, which differs between populations in its prevalence, morbidity, mortality, management success, adverse effects, and treatment options. The authors of asthma clinical trials that we reviewed appeared to assume that if a clinical trial was conducted in several countries or upon several racial/ethnic groups then the effects of the interventions would by default be valid in all the countries and racial/ethnic groups. This assumption (an example of the 'ecological fallacy', or 'biased sampling') requires that clinical outcomes were statistically and clinically homogeneous among the countries and racial/ethnic groups. However, consistently homogeneous outcomes seem rather unlikely, in light of the widely reported differences in asthma outcomes between populations (Supplementary Tables S1 and S2). High-profile studies such as 
SMART (the Salmeterol Multicenter Asthma Research Trial) have also highlighted how a lack of relevant genetic information for trial participants can hinder understanding, interpretation, and generalisation of adverse events resulting from asthma management interventions (Lipworth, 2007).

A consistent feature of the papers that we reviewed is that they lacked advice for clinicians about the generalizability of their findings, and none provided any guidance to assist clinicians to interpret the racial/ethnic applicability of treatment interventions in a patient-centred way. It is unclear, for example, whether the fact that Black patients always made up less than $23 \%$ of the study populations would limit the applicability of the trial findings to an individual Black patient. Despite the well documented evidence base for racial/ethnic, socio-economic, and genetic differences in asthma, none of the randomized controlled trials provided any formal hypotheses that incorporated any of these demographic variables. The trials, therefore, do not link clearly to the demographic evidence base and would not contribute to demographic healthcare goals, for example the reduction or elimination of demographic disparities in asthma. For the majority of the asthma trials that stated the race/ethnicity of their populations (17/23), it is unclear why multiple racial/ethnic groups were considered important enough to be included in the trial, yet were not separated when analyzing and interpreting outcomes. We assume that the function of the baseline race/ethnicity data in these trials was to demonstrate comparability of the racial/ethnic population mix across interventions, rather than to investigate effects of the population mix per se. However, to avoid committing the ecological fallacy (incorrectly applying aggregate group response data to all individual participants or subgroups, such as Black or White patients), prospective trials would either have to be restricted to homogeneous populations, sometimes called 'ethnic specific' trials, or should include appropriate analyses of population sub-groups. The choice of method (ethnic-specific or sub-group analyses) is a matter of debate (Cohn, 2006). Ethnic-specific trials have been supported by some authors (Taylor \& Wright, 2005) but have also been strongly criticized (Cooper \& Psaty, 2005) as "a step backwards" on account of their limited generalizability, limited prior evidence to justify the selection of a single study population, and absence of clinical rationale. Sub-group analyses would need to be defined a priori, adequately powered, and based on stratified sampling of the populations in order to detect population differences (Yusuf, Wittes, Probstfield, \& Tyroler, 1991). To inform the choice of analysis approach, it is first necessary to identify whether and where the representation of minorities in samples matters, for example whether race/ethnicity is a causal, mediating or nuisance variable (Allmark, 2004; Kraemer \& Wilson, 2002). As the representation of minorities in clinical trials will have costs (more patients need to be recruited to ensure adequate statistical power of sub-group analyses), it has been proposed that cost-benefit analysis should be part of the decision making process and this should be reported when justifying the selection and analysis of populations in clinical trials (Allmark, 2004). 
There have been extensive recommendations and requirements for the improved representation of racial/ethnic minorities in clinical trials, especially in the US (Sheikh et al., 2004), but these have not specifically addressed the issues mentioned above about why and how the minority populations should be selected, included and analyzed. We suspect that the vagueness of the guidance might be at least partly responsible for the limited attention to minority populations in asthma clinical trials. We acknowledge that ongoing debates over the merits of ethnic-specific versus sub-group analyses might make it difficult to develop very specific guidance on how minority populations should be analyzed in clinical trials, but the guidance should at least emphasize the need for transparent justification of whichever approaches are used for the selection, inclusion, and analysis of minority populations. Such justification should be formulated in clear hypotheses that link to the existing demographic and clinical bases of evidence and also to specific healthcare goals.

\section{Regulatory aspects - and contradictions}

Extrapolation of the results of clinical trials from a 'foreign' geographical region to a 'new' region has been discussed by the International Conference on Harmonisation of Technical Requirements for Registration of Pharmaceuticals for Human Use (ICH) under the auspices of the European Medicines Agency (EMEA) (European Medicines Agency, 1998). The ICH advises that for registration of medicines in the three ICH regions (Europe, the US, and Japan), it is critical to evaluate data in the major 'racial' groups most relevant to these regions, namely Caucasian, Black and Asian. However, this guidance appears to have ignored the criticism that such broad racial/ethnic groups lack biological relevance. Recent guidance from EMEA (European Medicines Agency, 2006) mentions that 'race' should usually be reported in clinical studies and, ideally, analyzed quantitatively. However, 'race', unlike 'ethnicity' (European Medicines Agency, 1998), is not explicitly defined by EMEA. The guidance (European Medicines Agency, 1996) also states that multi-centre studies should report results for individual centres and the comparability of centres should be assessed if possible, although our findings provide no evidence that such information from randomized controlled trials has reached the peer-reviewed literature. The European guidance seems to suffer not only from inconsistency with recommendations in the literature but also exhibits the problem that we refer to above - that it may be too vague to elicit specific action in terms of establishing how and why minorities should be included and analyzed in clinical trials.

\section{An important leap: from clinical study reports to the public domain}

Health regulatory agencies require more detailed information on clinical studies than would be feasible to report in published articles of the type included in our systematic review. For example, 
EMEA require detailed data for each of the patients involved in a clinical study (European Medicines Agency, 1996), and regulatory dossiers supplied by drug manufacturers may contain thousands of pages of information. Our review may, therefore, have underrepresented the extent to which data on race/ethnicity and socio-economic variables have been collected and analyzed. Nevertheless, the published literature reflects the majority of data accessible to clinicians, patients and researchers, whereas unpublished data from clinical trials are usually considered commercially confidential and effectively inaccessible. Strategic plans by the US National Institutes of Health (National Institutes of Health, 2004) and others to increase the representation of 'minority and ethnic populations' in clinical trials are unlikely to assist researchers and policymakers if the data they stimulate remain confined to clinical study reports and regulatory dossiers. Our review identifies a clear need to improve the transfer of such information into the public domain, underlining recommendations made by the World Health Organization that research findings "should not only be accessible to decision-makers but also communicated in ways that effectively inform policy, public health and health care decision-making', (World Health Organization, 2005).

\section{Recommendations}

Guidance on the conduct of asthma clinical trials should encourage more critical consideration and clearer reporting of how and why populations are selected and analyzed. Current guidance that merely recommends or requires that minorities are included in clinical trials, without requiring an explanation of how or why is unlikely to result in the generation of evidence relevant to demographic healthcare goals such as reducing or eliminating healthcare disparities in asthma. There is also a need for more critical consideration of the generalizability of clinical trial findings. Ideally, population analyses should be based on clearly specified hypotheses that link both to the existing demographic and clinical evidence and to demographic and clinical healthcare goals. Guidance should also stress the need for more precise reporting of demographic characteristics and genetic information, in order to reduce the subjectivity of describing and interpreting population classifications; to distance research trials from political sensitivities associated with race and ethnicity and from debates over the relative importance of genetic and environmental factors; and to facilitate a clearer understanding of the generalizability of trial findings.

\section{References}

Agyemang, C. (2006). Ethnic misclassifications hamper progress in research. BMJ, $332,1335$.

Agyemang, C., Bhopal, R., \& Bruijnzeels, M. (2005). Negro, Black, Black African, African 
Caribbean, African American or what? Labelling African origin populations in the health arena in the 21 st century. Journal of Epidemiology and Community Health, $59,1014-1018$.

Aldhous, P. (2002). Geneticist fears 'race neutral' studies will fail ethnic groups. Nature, 418, 355-356.

Allmark, P. (2004). Should research samples reflect the diversity of the population? Journal of Medical Ethics, 30, 185-189.

Almqvist, C., Worm, M., \& Leynaert, B. (2008). Impact of gender on asthma in childhood and adolescence: a GA2LEN review. Allergy, 63, 47-57.

American Anthropological Association. (1997). American anthropological association response to OMB directive 15: Race and ethnic standards for federal statistics and administrative reporting. http://www.aaanet.org/gvt/ombdraft. htm. Accessed July 2008.

Anonymous. (1996). Ethnicity, race and culture: guidelines for research, audit and publication. BMJ, 312, 1054.Anonymous. (2000). Census, race and science. Nature Genetics, 24, 97-98.

Apter, A. J. (2006). The influence of health disparities on individual patient outcomes: what is the link between genes and environment? Journal of Allergy and Clinical Immunology, 117, 345-350.

Bateman, E. D., Hurd, S. S., Barnes, P. J., Bousquet, J., Drazen, J. M., FitzGerald, M., et al. (2008). Global strategy for asthma management and prevention: GINA executive summary. European Respiratory Journal, 31, 143-178.

de Benedictis, F. M., Baraldi, E., \& Boner, A. (2008). Gender differences in the effectiveness of asthma treatment. Pediatrics, 121, 1289-1290.

Bhopal, R. (2004). Glossary of terms relating to ethnicity and race: for reflection and debate. Journal of Epidemiology and Community Health, 58, 441-445.

Bhopal, R., \& Donaldson, L. (1998). White, European, Western, Caucasian, or what? 
Inappropriate labeling in research on race, ethnicity and health. American Journal of Public Health, 88, 1303-1307.

Bhopal, R. S., Phillimore, P., \& Kohli, H. S. (1991). Inappropriate use of the term 'Asian': an obstacle to ethnicity and health research. Journal of Public Health Medicine, 13, 244-246.

Brahan, D., \& Bauchner, H. (2005). Changes in reporting of race/ethnicity, socioeconomic status, gender, and age over 10 years. Pediatrics, 115, e163-e166.

Choudhry, S., Ung, N., Avila, P. C., Ziv, E., Nazario, S., Casal, J., et al. (2005).

Pharmacogenetic differences in response to albuterol between Puerto Ricans and Mexicans with asthma. American Journal of Respiratory and Critical Care Medicine, 171, 563-570.

Cohn, J. N. (2006). The use of race and ethnicity in medicine: lessons from the African-American Heart Failure Trial. Journal of Law, Medicine and Ethics, 34, $552-554$

Collins, F. S. (2004). What we do and don't know about 'race', 'ethnicity', genetics and health at the dawn of the genome era. Nature Genetics, 36(Suppl. 11), S13-S15.

Committee on Understanding and Eliminating Racial and Ethnic Disparities in Health Care. (2003). Unequal treatment: Racial and ethnic disparities in health care. Washington, DC: National Academies Press.

Coons, S. J. (2006). Reporting race and ethnicity in clinical studies and health services research. Clinical Therapeutics, 28, 430-431.

Cooper, R. S. (2003). Gene-environment interactions and the etiology of common complex disease. Annals of Internal Medicine, 139, 437-440.

Cooper, R. S., Kaufman, J. S., \& Ward, R. (2003). Race and genomics. New England Journal of Medicine, 348, 1166-1170.

Cooper, R. S., \& Psaty, B. M. (2005). Diversity and inclusiveness should remain the guiding principles for clinical trials. Circulation, 112, 3660-3666. 
Corbie-Smith, G., St George, D. M. M., Moody-Ayers, S., \& Ransohoff, D. F. (2003). Adequacy of reporting race/ethnicity in clinical trials in areas of health disparities. Journal of Clinical Epidemiology, 56, 416-420.

Diette, G. B., \& Rand, C. (2007). The contributing role of health-care communication to health disparities for minority patients with asthma. Chest, 132, 802S-809S.

Dorne, J. L. C. M. (2007). Human variability in hepatic and renal elimination: implications for risk assessment. Journal of Applied Toxicology, 27, 411-420.

European Medicines Agency (EMEA). (1996). Structure and content of clinical study reports. ICH Topic E3. Report CPMP/ICH/137/95. London: European Medicines Agency.

European Medicines Agency (EMEA). (1998). Note for guidance on ethnic factors in the acceptability of foreign clinical data. ICH Topic E5 (R1). Report CPMP/ICH/289/ 95. London: European Medicines Agency.

European Medicines Agency (EMEA). (2006). Ethnic factors in the acceptability of foreign clinical data. Questions and answers. ICH Topic E5 (R1). Report CPMP/ICH/ 5746/03. London: European Medicines Agency.

Gonza' lez Buchard, E., Borrell, L. N., Choudhry, S., Navqi, M., Tsai, H. J., RodriguezSantana, J. R., et al. (2005). Latino populations: a unique opportunity for the study of race, genetics, and social environment in epidemiological research. American Journal of Public Health, 95, 2161-2168.

Hall, I. P., \& Sayers, I. (2007). Pharmacogenetics and asthma: false hope or new dawn? European Respiratory Journal, 29, 1239-1245.

Holguin, F., Mannino, D. M., Anto, J., Mott, J., Ford, E. S., Teague, W. G., et al. (2005). Country of birth as a risk factor for asthma among Mexican Americans. American Journal of Respiratory and Critical Care Medicine, 171, 103-108.

Hussain-Gambles, M., Atkin, K., \& Leese, B. (2004). Why ethnic minority groups are under-represented in clinical trials: a review of the literature. Health and Social 
Care in the Community, 12, 382-388.

Huth, E. J. (1995). Identifying ethnicity in medical papers. Annals of Internal Medicine, $122,619-621$.

International Committee of Medical Journal Editors. (1997). Uniform requirements for manuscripts submitted to biomedical journals. Annals of Internal Medicine, $126,36-47$.

Ishizuka, T., Hisada, T., Aoki, H., Yanagitani, N., Kaira, K., Utsugi, M., et al. (2007).

Gender and age risks for hoarseness and dysphonia with use of a dry powder fluticasone propionate inhaler in asthma. Allergy and Asthma Proceedings, 28, $550-556$.

Israel, E., Chinchilli, V. M., Ford, J. G., Boushey, H. A., Cherniack, R. M., Craig, T. J., et al. (2004). Use of regularly scheduled albuterol treatment in asthma: genotypestratified, randomised, placebo-controlled cross-over trial. Lancet, 364, $1505-1512$.

Israel, E., Drazen, J. M., Liggett, S. B., Boushey, H. A., Cherniack, R. M., Chinchilli, V. M., et al. (2000). The effect of polymorphisms of the B2-adrenergic receptor on the response to regular use of albuterol in asthma. American Journal of Respiratory and Critical Care Medicine, 162, 75-80.

Jensen-Jarolim, E., \& Untersmayr, E. (2008). Gender-medicine aspects in allergology. Allergy, 63, 610-615.

Johnston, N.W., Mandhane, P. J., Dai, J., Duncan, J. M., Greene, J. M., Lambert, K., et al. (2007). Attenuation of the September epidemic of asthma exacerbations in children: a randomized, controlled trial of montelukast added to usual therapy. Pediatrics, 120, e702-e712.

Johnston, N. W., \& Sears, M. R. (2006). Asthma exacerbations. 1: epidemiology. Thorax, 61, 722-728.

Kaplan, J. B., \& Bennett, T. (2003). Use of race and ethnicity in biomedical publication. JAMA, 289, 2709-2716. 
Kaufman, J. S., \& Cooper, R. S. (2001). Commentary: considerations for use of racial/ ethnic classification in etiologic research. American Journal of Epidemiology, 154, 291-298.

Koysooko, R., Tuchinda, M., Habananada, S., Pinthong, T., Wattanapermpool, J., \& Geadsomnuig, S. (1987). Pharmacokinetics of oral theophylline in Thai asthmatic children. Asian Pacific Journal of Allergy and Immunology, 5, 179-185.

Kraemer, H. C., \& Wilson, G. T. (2002). Mediators and moderators of treatment effects in randomized controlled trials. Archives of General Psychiatry, 59, 877-883.

Kuehni, C. E., Strippoli, M. P., Low, N., \& Silverman, M. (2007). Asthma in young south Asian women living in the United Kingdom: the importance of early life. Clinical and Experimental Allergy, 37, 47-53.

Leung, R. C., Carlin, J. B., Burdon, J. G., \& Czarny, D. (1994). Asthma, allergy and atopy in Asian immigrants in Melbourne. The Medical Journal of Australia, 161, 418-425. Lipworth, B. (2007). Long-acting beta-2-adrenoceptor agonists: a smart choice for asthma? Trends in Pharmacological Sciences, 28, 257-262.

Ma, I. W. Y., Khan, N. A., Kang, A., Zalunardo, N., \& Palepu, A. (2007). Systematic review identified suboptimal reporting and use of race/ethnicity in general medical journals. Journal of Clinical Epidemiology, 60, 572-578.

Main, C., Shepherd, J., Anderson, R., Rogers, G., Thompson-Coon, J., Liu, Z., et al. (2008). Systematic review and economic analysis of the comparative effectiveness of different inhaled corticosteroids and their usage with long-acting beta-2 agonists for the treatment of chronic asthma in children under the age of 12 years. Health Technology Assessment, 12(19), 1-192.

Maxwell, T. J., Ameyaw, M. M., Pritchard, S., Thornton, N., Folayon, G., Githang'a, J., et al. (2005). Beta-2 adrenergic receptor genotypes and haplotypes in different ethnic groups. International Journal of Molecular Medicine, 16, 573-580.

Migliore, E., Pearce, N., Bugiani, M., Galletti, G., Biggieri, A., Bisanti, L., et al. (2007). Prevalence of respiratory symptoms in migrant children to Italy: the results of SIDRIA-2 study. Allergy, 62, 293-300. 
Moher, D., Schultz, K. F., \& Altman, D. G. (2001). The CONSORT statement: revised recommendations for improving the quality of reports of parallel-group randomized controlled trials. Lancet, 357, 1191-1194.

Munakata, M., Harada, Y., Ishida, T., Saito, J., Nagabukuro, A., Matsushita, H., et al. (2006). Molecular-based haplotype analysis of the beta 2-adrenergic receptor gene (ADRB2) in Japanese asthmatic and non-asthmatic subjects. Allergology International, 55, 191-198.

National Institutes of Health. (2004). Strategic research plan and budget to reduce and ultimately eliminate health disparities, Vol. I. Bethesda, MD: National Institutes of Health.

Nave, R., Fisher, R., \& McCracken, N. In vitro metabolism of beclomethasone dipropionate, budesonide, ciclesonide, and fluticasone propionate in human lung precision-cut tissue slices. Respiratory Research, in press.

Netuveli, G., Hurwitz, B., Levy, M., Fletcher, M., Barnes, G., Durham, S. R., et al. (2005). Ethnic variations in UK asthma frequency, morbidity and health-service use: a systematic review and meta-analysis. Lancet, 365, 312-317.

Pearce, R. E., Leeder, J. S., \& Kearns, G. L. (2006). Biotransformation of fluticasone: in vitro characterization. Drug Metabolism and Disposition, 34, 1035-1040.

Peet, C. F., Enos, T., Nave, R., Zech, K., \& Hall, M. (2005). Identification of enzymes involved in phase I metabolism of ciclesonide by human liver microsomes. European Journal of Drug Metabolism and Pharmacokinetics, 30, 275-286.

Rivara, F., \& Finberg, L. (2001). Use of the terms race and ethnicity. Archives of Pediatrics and Adolescent Medicine, 155, 119.

Salari, K., Choudhry, S., Tang, H., Naqvi, M., Lind, D., Avila, P. C., et al. (2005). Genetic admixture and asthma-related phenotypes in Mexican American and Puerto Rican asthmatics. Genetic Epidemiology, 21, 76-86.

Sayers, I., \& Hall, I. P. (2007). Pharmacogenetic approaches in the treatment of 
asthma. Current Allergy and Asthma Reports, 5, 101-108.

Schwartz, R. S. (2001). Racial profiling in medical research. New England Journal of Medicine, 344, 1392-1393.

Senior, P. A., \& Bhopal, R. (1994). Ethnicity as a variable in epidemiological research. BMJ, 309, 327-330.

Shanawani, H. (2006). Health disparities and differences in asthma: concepts and controversies. Clinics in Chest Medicine, 27, 17-28.

Shanawani, H., Dame, L., Schwartz, D. A., \& Cook-Deegan, R. (2006). Non-reporting and inconsistent reporting of race and ethnicity in articles that claim associations among genotype, outcome, and race or ethnicity. Journal of Medical Ethics, 32, 724-728.

Sheikh, A., Panesar, S. S., Lasserson, T., \& Netuveli, G. (2004). Recruitment of ethnic minorities to asthma studies. Thorax, 59, 634 .

Shepherd, J., Rogers, G., Anderson, R., Main, C., Thompson-Coon, J., Hartwell, D., et al. (2008). Systematic review and economic analysis of the comparative effectiveness of different inhaled corticosteroids and their usage with longacting beta- 2 agonists for the treatment of chronic asthma in adults and children aged 12 years and over. Health Technology Assessment, 12(20), 1-274.

Smart, A., Tutton, R., Martin, P., Ellison, G. T. H., \& Ashcroft, R. (2008). The standardization of race and ethnicity in biomedical science editorials and UK biobanks.

Social Studies of Science, 38, 407-423.

Szefler, S. J., Phillips, B. R., Martinez, F. D., Chinchilli, V. M., Lemanske, R. F., Strunk, R. C., et al. (2005). Characterization of within-subject responses to fluticasone and montelukast in childhood asthma. Journal of Allergy and Clinical Immunology, 115, 233-242.

Taylor, A. L., \& Wright, J. T. (2005). Importance of race/ethnicity in clinical trials. Circulation, 112, 3654-3660.

Taylor, D. R., Drazen, J. M., Herbison, G. P., Yandava, C. N., Hancox, R. J., \& Town, G. I. 
(2000). Asthma exacerbations during long term $\beta$ agonist use: influence of $\beta 2$ adrenoceptor polymorphism. Thorax, 55, 762-767.

Tedeschi, A., Barcella, M., Bo, G. A., \& Miadonna, A. (2003). Onset of allergy and asthma symptoms in extra-European immigrants to Milan, Italy: possible role of environmental factors. Clinical and Experimental Allergy, 33, 449-454.

Tonorezos, E. S., Breysse, P. N., Matsui, E. C., McCormack, M. C., Curtin-Brosnan, J., Williams, D., et al. (2008). Does neighbourhood violence lead to depression among caregivers of children with asthma? Social Science \& Medicine, 67, 31-37.

UK National Institute for Health and Clinical Excellence. (2008a). Corticosteroids for the treatment of chronic asthma in adults and children aged 12 years and over. NICE Technology Appraisal Report 138. London: National Institute for Health and Clinical Excellence. http://www.nice.org.uk/TA138. Accessed July 2008.

UK National Institute for Health and Clinical Excellence. (2008b). Inhaled corticosteroids for the treatment of chronic asthma in children under the age of 12 years.

NICE Technology Appraisal Report 131. London: National Institute for Health and Clinical Excellence. http://www.nice.org.uk/TA131. Accessed July 2008.

Walsh, C., \& Ross, L. F. (2003). Whether and why pediatric researchers report race and ethnicity. Archives of Pediatrics and Adolescent Medicine, 157, 671-675.

Wechsler, M. E., Lehman, E., Lazarus, S. C., Lemanske, R. F., Boushey, H. A., Deykin, A., et al. (2006). Beta-adrenergic receptor polymorphisms and response to salmeterol. American Journal of Respiratory and Critical Care Medicine, 173, $519-526$.

Winker, M. A. (2004). Measuring race and ethnicity: why and how? JAMA, 292, $1612-1614$

Witzig, R. (1996). The medicalization of race: scientific legitimization of a flawed social construct. Annals of Internal Medicine, 125, 675-679.

World Health Organization. (2005). Ministerial summit on health research (Mexico City, 16-20 November 2004). Geneva: World Health Organization. http://www. 
who.int/rpc/summit/en. Accessed May 2008.

Xie, H. G., Stein, C. M., Kim, R. B., Xiao, Z. S., He, N., Zhou, H. H., et al. (1999).

Frequency of functionally important beta-2 adrenoceptor polymorphisms varies

markedly among African-American, Caucasian and Chinese individuals.

Pharmacogenetics, 9, 511-516.

Yusuf, S., Wittes, J., Probstfield, J., \& Tyroler, H. A. (1991). Analysis and interpretation of treatment effects in subgroups of patients in randomized clinical trials. JAMA, $266,93-98$.

\section{Figure legends}

Figure 1. QUOROM chart summarizing the provenance of published papers included in the systematic review.

Figure 2. Temporal distribution of randomized controlled trials (RCTs)

included in the systematic review. 


\section{Figure 1}

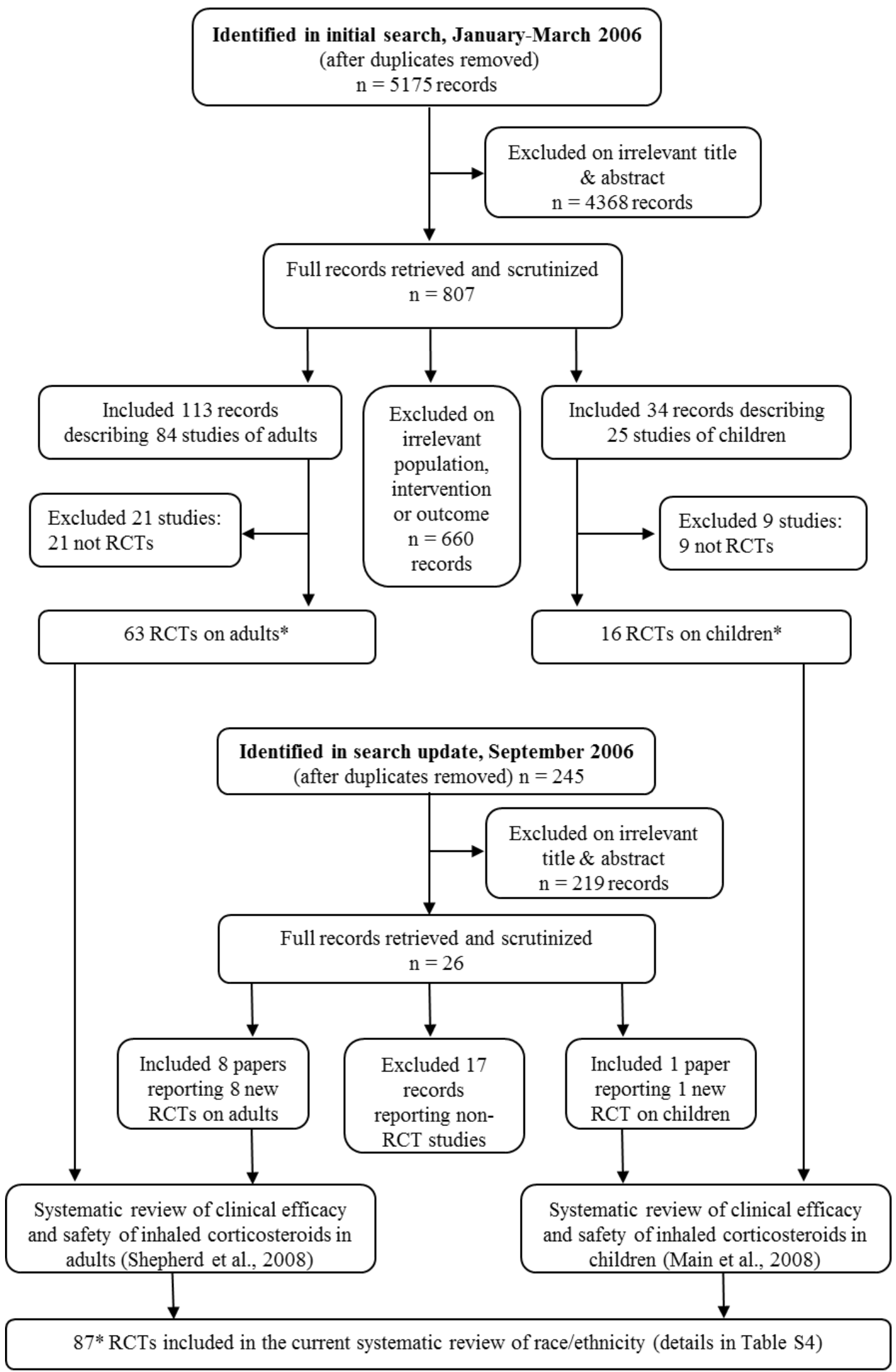

* One RCT included both adults and children 
Figure 2

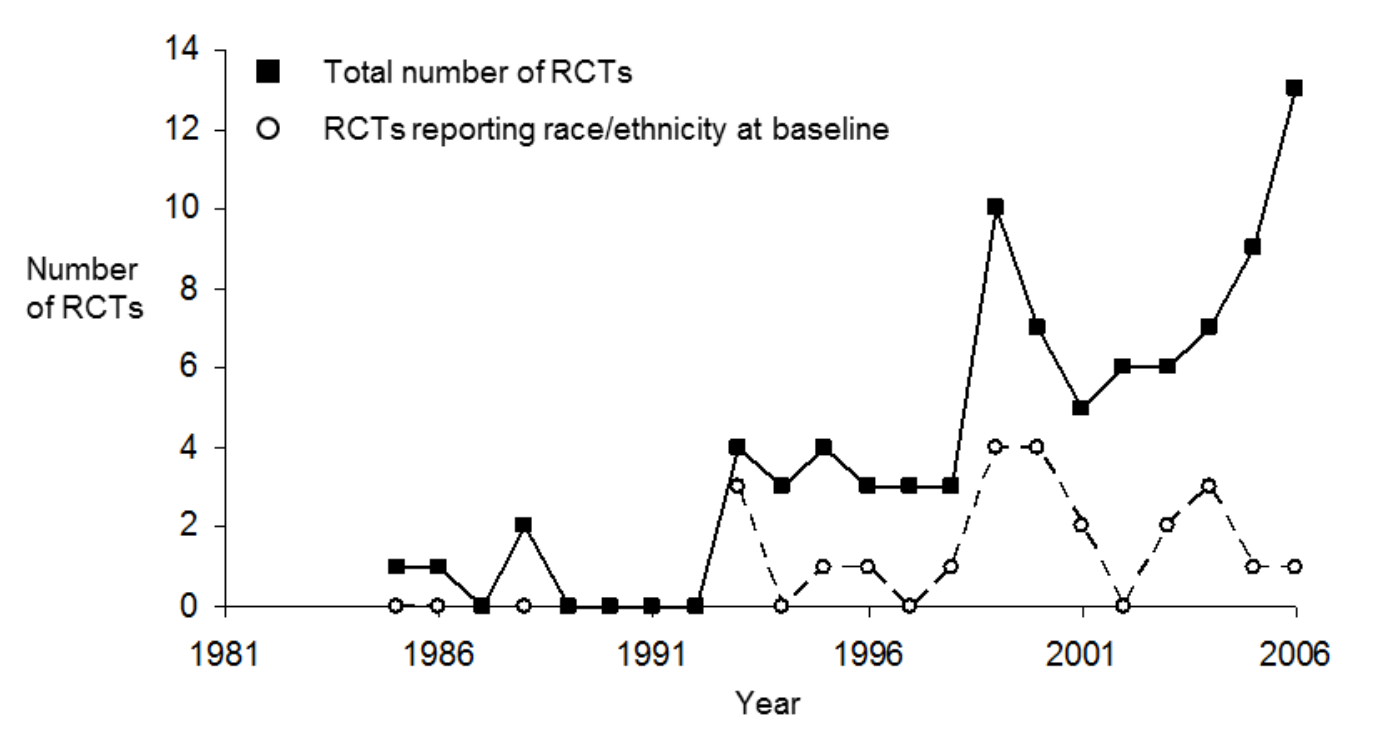

